\title{
CORRESPONDENCE
}

\section{Intraprostatic Pacinian corpuscle does exist!}

\section{To the Editor,}

Pacinian corpuscle is a low threshold mechanoreceptor involved in the sensation of pressure, touch, and vibration. Its structure comprises a capsule, flat cells (Schwann-like glial cells, or lamellar cells) arranged in a concentric pattern of about 30 lamellae and a central core with an axon terminal. ${ }^{1,2}$ Pacinian corpuscles are commonly seen in dermis and subcutaneous tissues of the hands and feet and the digits, arm, neck, mesenteries, near joints, nipples, external genitalia, and periosteal and interosseous membranes. ${ }^{3}$ They are exceptionally rare in other locations and have been documented in urinary bladder, pancreas, lymph nodes and thymus. ${ }^{4-7}$ They have also been observed in developmental disorders such as meningoradiculoceles and cervical chondrocutaneous rests. ${ }^{8,9}$ We are aware of two previous reports of Pacinian corpuscles in the prostate,${ }^{10,11}$ both of which were not in clear intraprostatic location.

We report herein the finding of a radical prostatectomy specimen from a 64-year-old patient. It showed a prostatic adenocarcinoma, Gleason score $9(4+5)$ (Grade group 5), with extraprostatic extension (pT3a). On microscopy, the periphery of the right apex showed-in addition to extensive prostatic malignancy-one bulbous onion-like formation measuring $1 \mathrm{~mm}$ (maximum diameter) in obvious intraprostatic location (Fig. 1). The corpuscle was located $0.5 \mathrm{~mm}$ deep from the external boundary of the prostate. Clinical history was unremarkable.

In the case reported by Medlicott and colleagues, two corpuscles were described as located in the periphery of mid- gland and, based on the photomicrograph, at least one of them was in the extraprostatic tissue. ${ }^{10}$ In the first report of prostatic Pacinian corpuscle, location and level of occurrence was not provided although the photomicrograph suggests the presence of adipose (extraprostatic) tissue surrounding the structure. $^{11}$ Extraprostatic tissue is known to harbour numerous and large paraganglia. In the bladder, Pacinian corpuscle can be seen within large paraganglia during the first months of life. In specimens of children older than 5 months, these structures are only seen separate. ${ }^{12}$ This observation suggests that paraganglia may be involved in the differentiation of Pacinian corpuscle during development. However, Pacinian corpuscles are exceptionally rare in the urinary bladder of adults.

We confirm the occurrence of Pacinian corpuscles within intraprostatic tissues (Fig. 1). In all three documented cases to date, Pacinian corpuscles were surrounded by high-grade invasive acinar adenocarcinoma. Awareness of variations of normal histology is important to avoid misinterpretation of unusual findings.

Conflicts of interest and sources of funding: The authors state that there are no conflicts of interest to disclose.

\section{Maiara Ferreira de Souza ${ }^{1}$, Daniel Abensur Athanazio ${ }^{1,2}$ \\ ${ }^{1}$ Imagepat, Laboratory of Pathology, Rio Vermelho, Salva- dor, BA, Brazil; ${ }^{2}$ Hospital Universitário Professor Edgard Santos, Federal University of Bahia, Salvador, BA, Brazil}

Contact Dr Daniel Athanazio.

E-mail: dathanazio@gmail.com
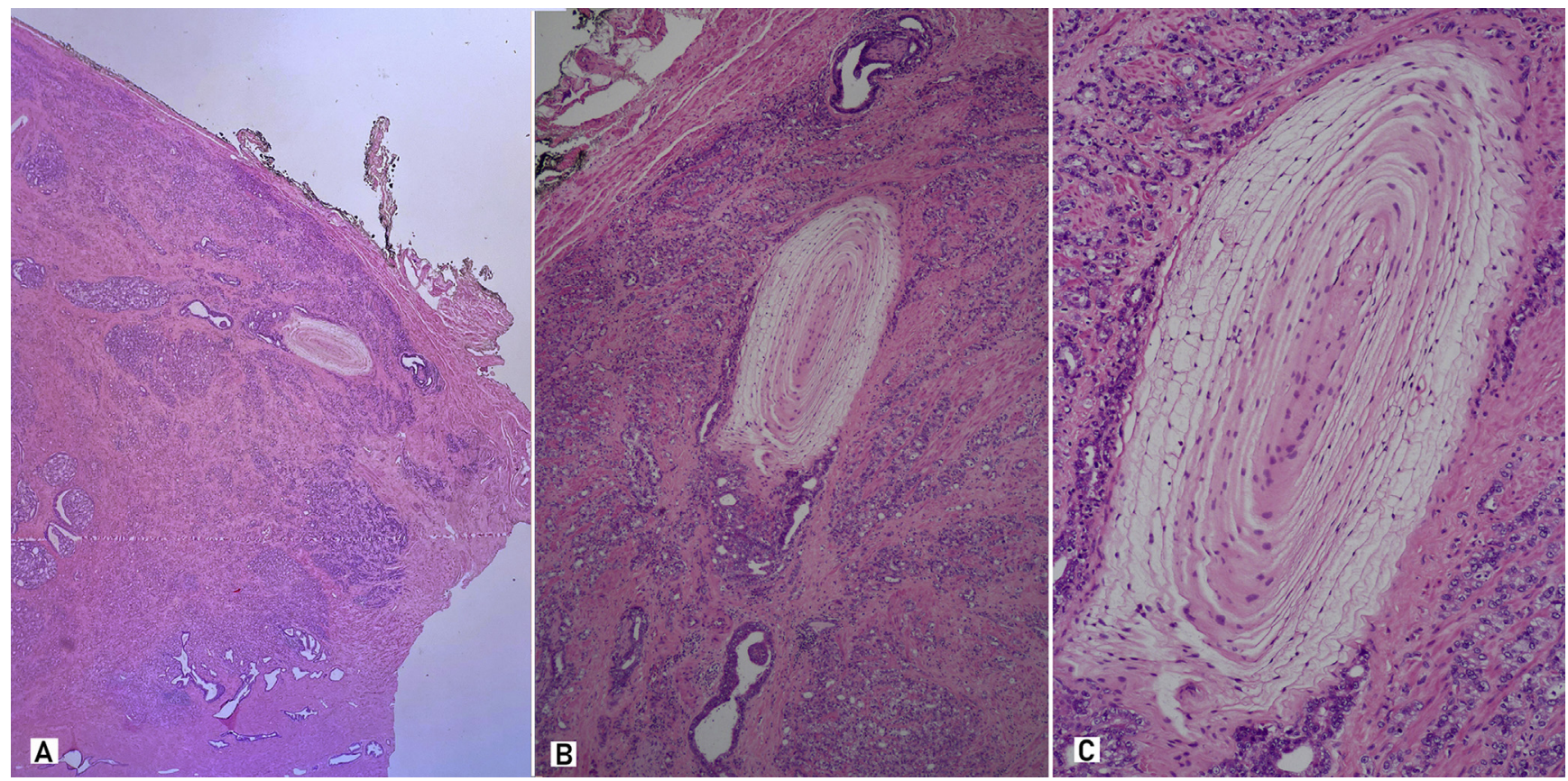

Fig. 1 Intraprostatic Pacinian corpuscle, H\&E. (A) Low power (B), higher power, (C) high power. Note a high-grade acinar adenocarcinoma in the surrounding prostatic tissues. 
1. Bentivoglio M, Pacini P. Filippo Pacini-a determined observer. Brain Res Bull 1995; 38: 161-5.

2. Cobo R, García-Piqueras J, Cobo J, Vega JA. The human cutaneous sensory corpuscles: an update. J Clin Med 2021; 10: 227.

3. Kettenmann H. Nervous system. In: Standring S, editor. Gray's Anatomy: The Anatomical Basis of Clinical Practice. 41st ed. Amsterdam: Elsevier, 2016; 61

4. Landon DN, Wiseman OJ. A Pacinian corpuscle in the human bladder lamina propria. J Neurocytol 2001; 30: 457-64.

5. Standop J, Ulrich A, Schneider MB, Andrén-Sandberg A, Pour PM. Pacinian corpuscle in the human pancreas. Pancreas 2001; 23: 36-9.

6. Aydin O. Pacinian corpuscle in a lymph node. Neuropathology 2006 26: $379-81$.

7. Varga I, Nosál M, Babál P. Ectopic lamellar Pacinian corpuscle within the thymus. Atypical or abnormal location? Rom J Morphol Embryol 2020; 61: 273-6.

8. Gessi M, Messing-Jünger M, Röhrig A, Gielen GH, Pietsch T, van Landeghem FK. Incidental neuropathological findings in a child with cervical meningoradiculocele. Brain Pathol 2012; 22: 737-40.

9. Feito J, Ramos-García JL, Gago Á, et al. Pacinian corpuscles in a cervical chondrocutaneous remnant: a case report and update of Pacinian corpuscles. Am J Dermatopathol 2016; 38: 231-5.

10. Pai SA. Ectopic Pacinian corpuscle in the prostate. Int J Surg Patho 2017: 25: 609-10.

11. Medlicott SAC, Larsen ET, Gao Y, Trpkov K. Pacinian corpuscle in the prostate: fact - not fiction! Hum Pathol Case Rep 2019; 15: 71-2.

12. Dixon JS, Gosling JA, Canning DA, Gearhart JP. An immunohistochemical study of human postnatal paraganglia associated with the urinary bladder. J Anat 1992; 181: 431-6.

DOI: https://doi.org/10.1016/j.pathol.2021.06.126

\section{An unusual testicular mass: a case of desmoplastic small round cell tumour}

To the Editor,

Desmoplastic small round cell tumour (DSRCT) is a rare, aggressive, mesenchymal malignancy of uncertain differentiation with both a characteristic chromosomal translocation and immunohistochemical profile. It most commonly presents with vague abdominal symptoms, most often in young, Caucasian males. Advanced disease on diagnosis with multiple intra-abdominal lesions is common. ${ }^{1}$ While typically an intra-abdominal malignancy, cases of DSRCT arising from lung, central nervous system, nasal sinus and paratesticular lesions have been reported rarely. ${ }^{2-4}$ Presentation as a primary testicular mass is exceedingly rare and poses a diagnostic challenge both in the clinical and pathological setting, as this tumour can mimic many other entities.

We present a case of a 29-year-old, otherwise well, male who presented with an enlarging right scrotal mass. An ultrasound demonstrated an abnormal, hypoechoic right testis measuring $6 \mathrm{~cm}$, concerning for a malignant mass replacing the testis. Unfortunately, he was non-compliant with medical advice and did not attend follow up for 3 months. Eventually, he attended an urgent urology appointment, and a computed tomography scan of the chest, abdomen and pelvis was performed. Imaging demonstrated multiple metastases including pulmonary lesions and diffuse lymphadenopathy in the left supraclavicular fossa, mediastinum and para-aortic regions measuring up to $6 \mathrm{~cm}$. Serum tumour markers were negative. Urgent admission to hospital was arranged, however the patient did not attend despite multiple attempts to facilitate treatment. Two months later, the patient presented to the emergency department requesting treatment. The suspected diagnosis was a testicular germ cell tumour with metastatic disease. Approximately $30 \%$ of patients with testis cancer have metastases at diagnosis.

Radical orchidectomy was performed and the patient recovered uneventfully post-operatively. Macroscopic examination showed a $75 \mathrm{~mm}$ testicular mass with tumour replacing the entire contents of the testis and epididymis (Fig. 1). The tumour was thoroughly sampled to reveal no gross or microscopic heterologous elements, suggestive of a postpubertal-type teratoma. Histologically, this tumour was composed of nests of malignant tumour surrounded by a desmoplastic stroma. The tumour cells had minimal cytoplasm and rounded to oval nuclei. Numerous mitoses were present with focal areas of single cell necrosis. There was extensive lymphovascular invasion with tumour deposits seen within the tunica vaginalis (Fig. 2). The cord contained continuous tumour that was present at the spermatic cord resection margin. Immunohistochemistry showed multiphenotypic differentiation with staining for epithelial membrane antigen (EMA), pancytokeratin (MNF-116), desmin (dot-like cytoplasmic), CD57, CD56 and neuron specific
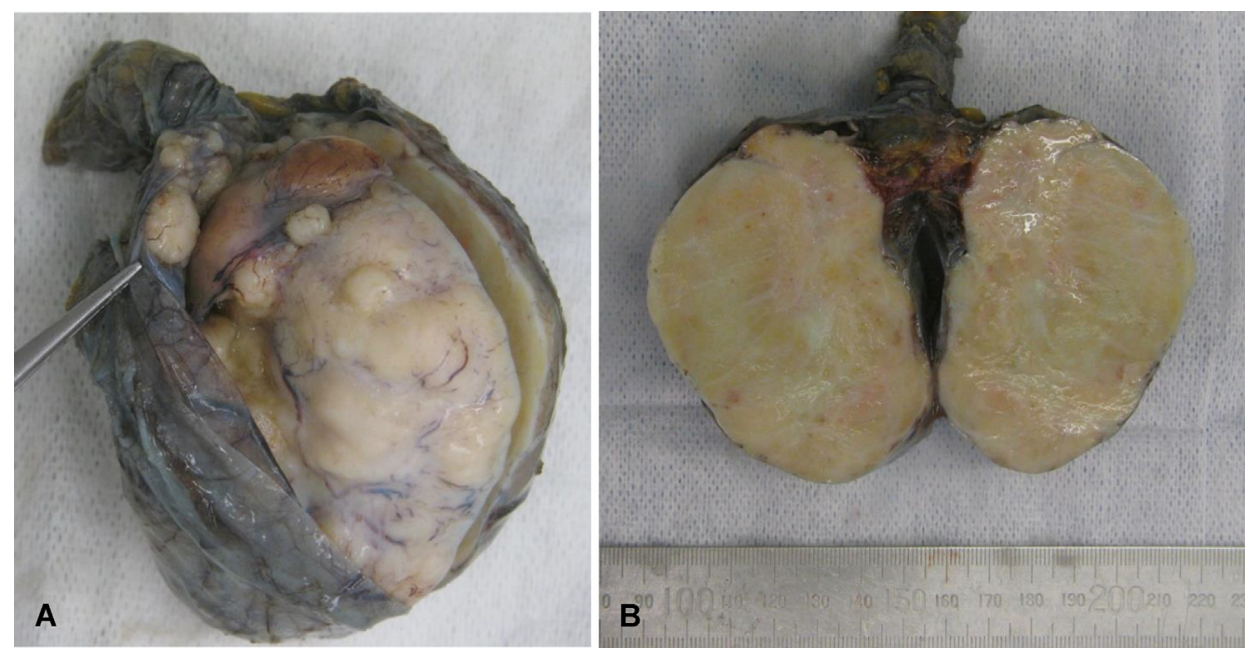

Fig. 1 Right radical orchidectomy specimen. (A) Right orchidectomy specimen with parietal layer of tunica vaginalis divided, demonstrating nodular testicular tumour with satellite nodules on tunica. (B) Bisected specimen demonstrating tan coloured tumour replacing entire contents of testis and epididymis. 